

\title{
la tribuna del hipódromo Yonkers
}

JACOB FELD, ingeniero LIONEL K. LEVY, arquitecto

La reforma del hipódromo Yonkers, de Nueva York, dedicado a las carreras de caballos de trote, a cuyas reuniones nocturnas suelen concurrir de 15 a 35.000 espectadores, se ha verificado siguiendo un programa desarrollado entre el tiempo muerto y las temporadas de actividad. Rodeado de otras edificaciones, el único espacio que se podía disponer era el ganado en el sentido vertical. Todo se encaminó, en primer lugar, hacia un acceso fácil y zonas cómodas de estacionamiento de vehículos. 
Colocación de las vigas de cubierta en el cuerpo principal.

Entramados provisionales o andamios.

Terminando la cubierta.

El estudio de esta reforma corrió a cargo del arquitecto Lionel $\mathbf{R}$. Levy, mientras que las estructuras y control general correspondió al Dr. Jacob Feld, que actuó como ingeniero de consulta.

La primera parte de la reforma consistió en sustituir las cuadras de madera por otras, de dos plantas, con estructura de hormigón y con capacidad para 600 caballos. La zona de estacionamiento se amplió de una capacidad de 1.140 coches a la de 7.400 .

La segunda parte fue suprimir el antiguo edificio de servicios y sustituirlo por otro nuevo, en el que se instalaría un restaurante y una terraza con mesas de bar y comedor con una capacidad para 4.000 asientos, cubierta de tal forma que no existiese obstáculo para poder ver toda la pista de carreras, de unos $800 \mathrm{~m}$ de longitud.

Para cubrir la tribuna superior, de una capacidad de 4.000 asientos, se proyectó una ménsula, de $61 \mathrm{~m}$ de longitud y $29 \mathrm{~m}$ en voladizo, que se creyó suficiente para proteger a los espectadores de las inclemencias del tiempo. La tribuna inferior tiene una serie escalonada de terrazas con mesas de restaurante y un comedor amplio. La planta baja constituye los pasos de circulación y de acceso al hipódromo.

En esta planta baja, en la segunda y una entreplanta, se hallan las taquillas de apuestas mutuas. Los distintos niveles se han unido por medio de escaleras mecánicas $\mathrm{y}$ ascensores.
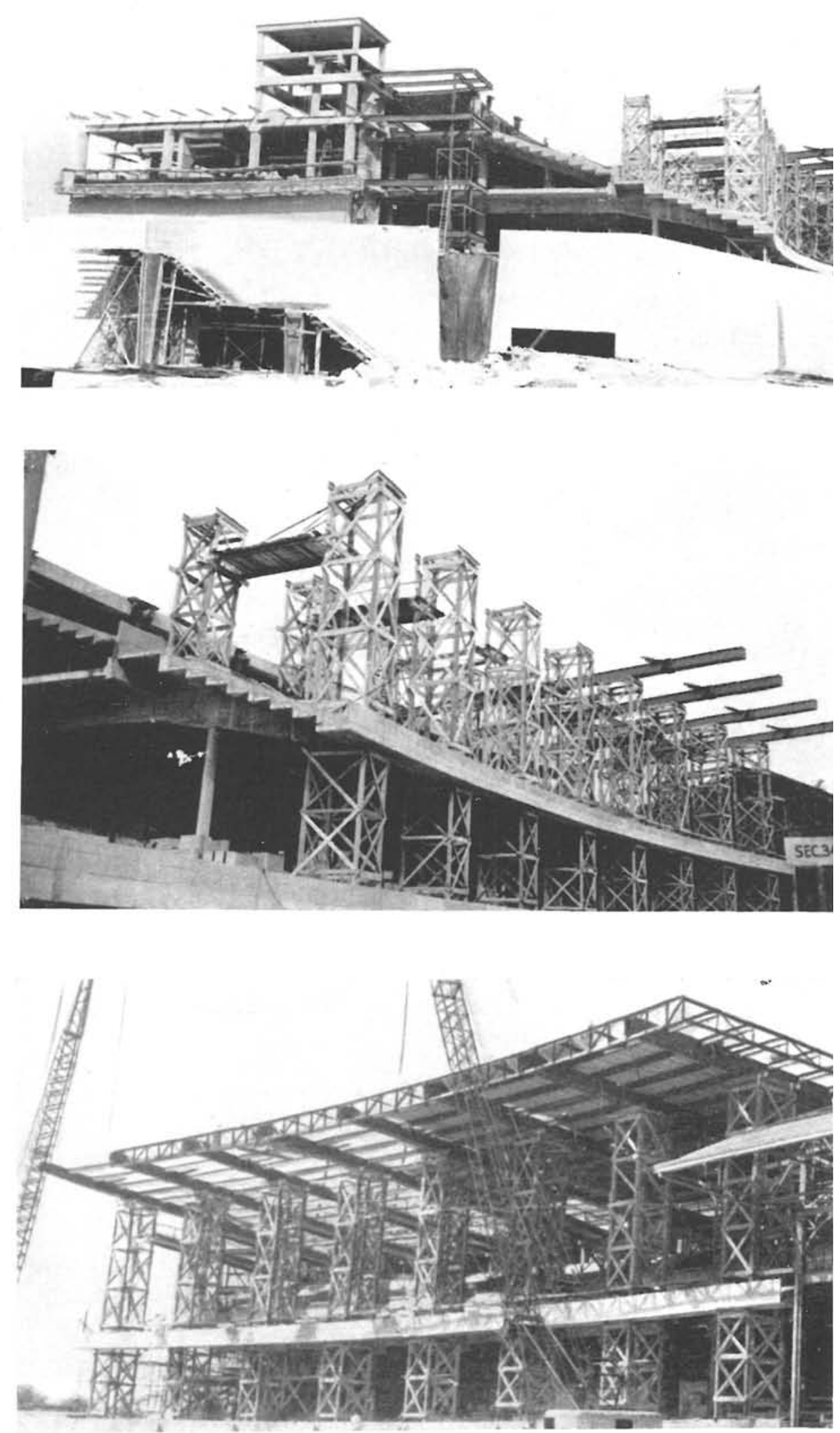


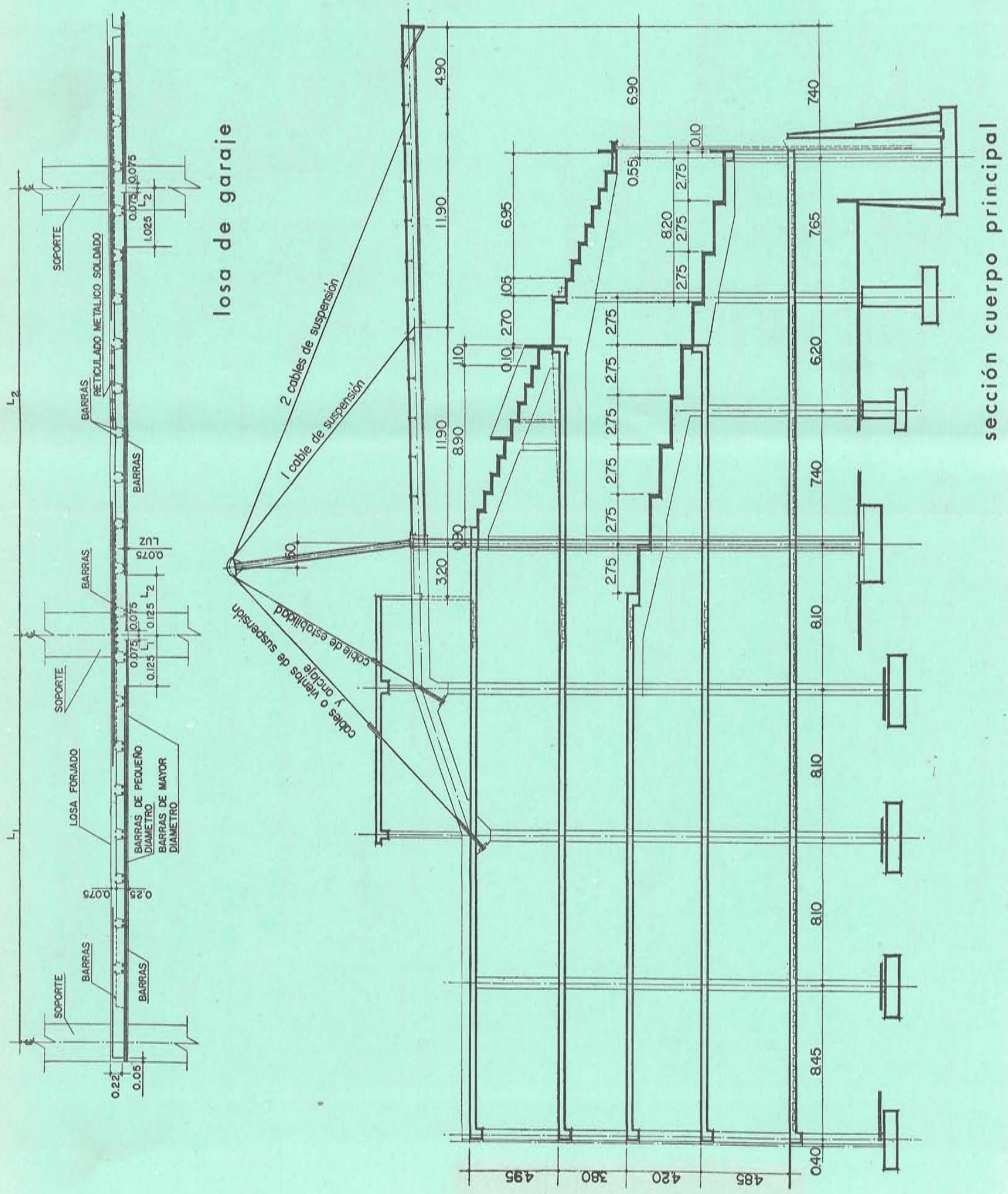



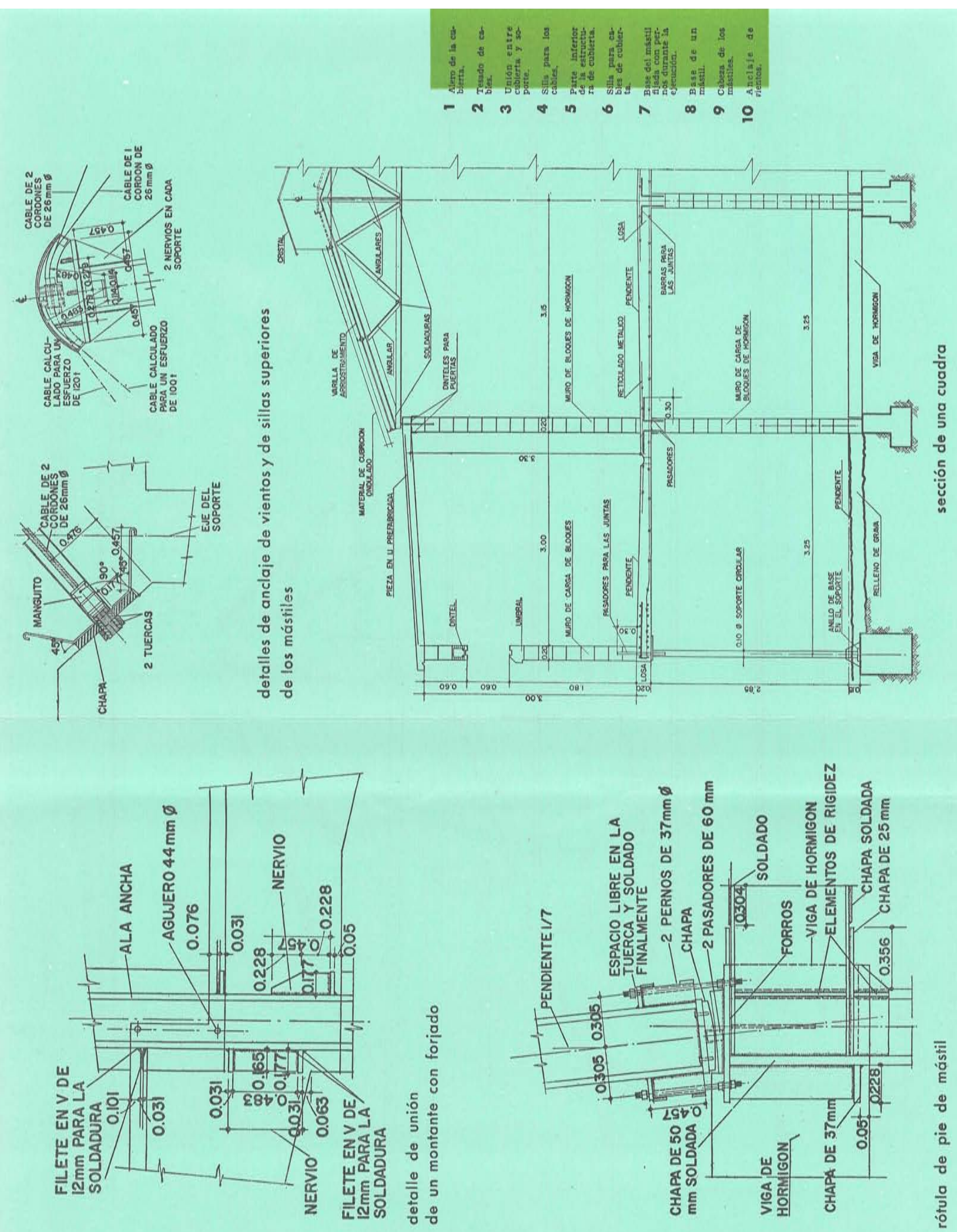

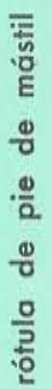



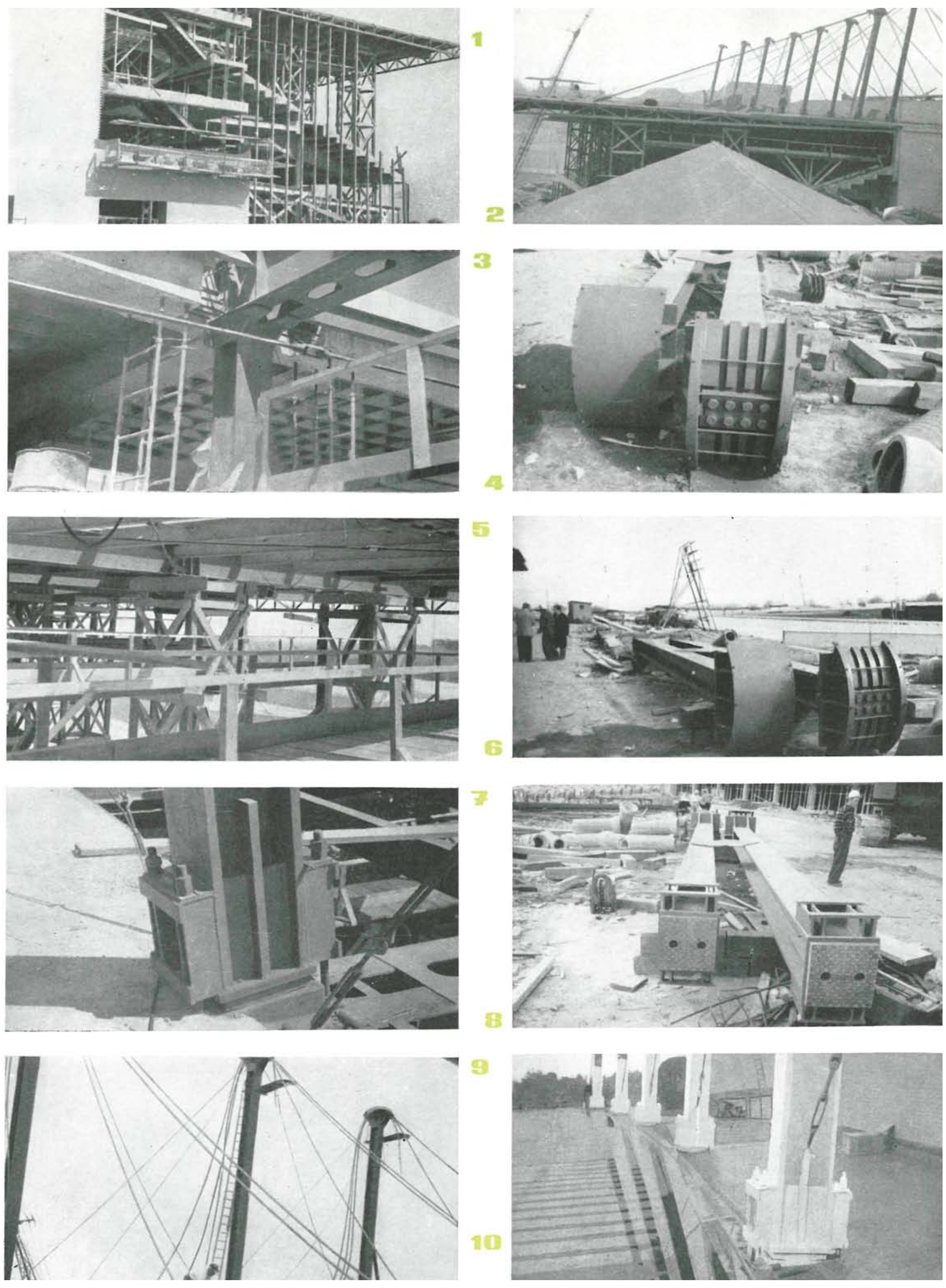


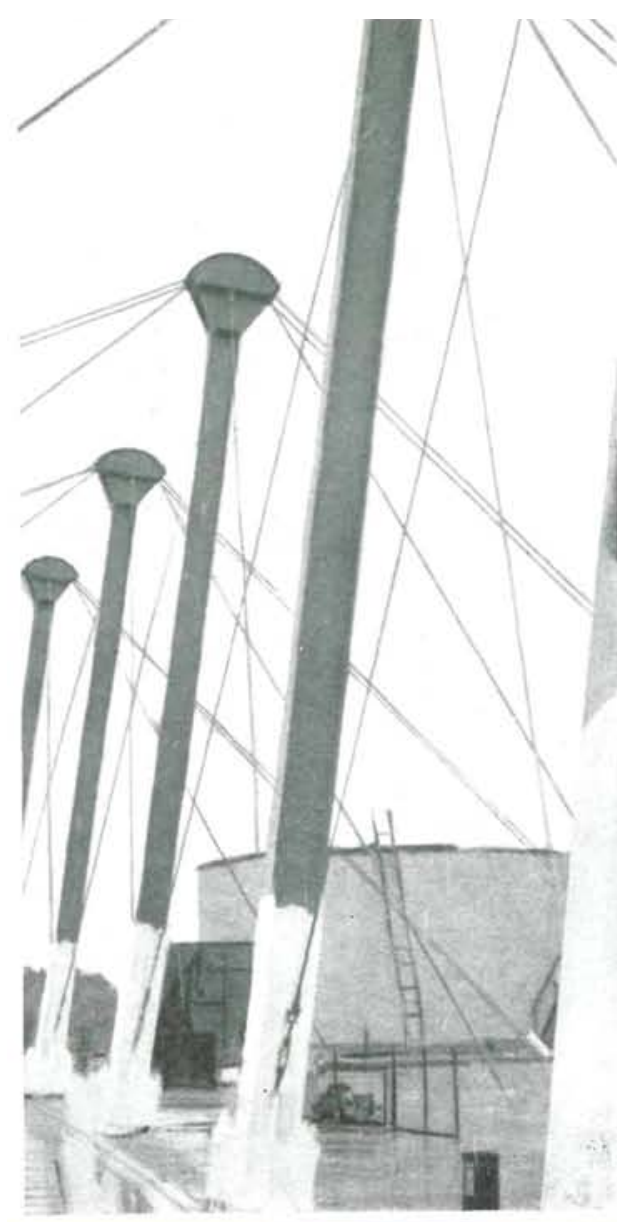

\section{La cubierta}

Los principios básicos de estabilidad son similares a los de los puentes suspendidos y la introducción de algunos elementos de hormigón pretensado.

En la parte posterior de la tribuna aparecen los soportes metálicos, espaciados a $8,50 \mathrm{~m}$, que se prolongan formando mástil de suspensión o torre. Estas torres tienen $10,60 \mathrm{~m}$ de altura, y de ellas parten los cables o vientos de suspensión que se unen a las vigas cajón, metálicas, en sus extremidades. Estos cables pasan sobre sillas colocadas en la parte superior de las torres y se prolongan hasta la parte superior del cuerpo del edificio central, donde se anclan.

Las sillas tienen un dispositivo de rótula, y las vigas que vuelan se apoyan en los soportes, que, inclinados hacia atrás, tienen una proyección horizontal de $1,50 \mathrm{~m}$. Entre las vigas suspendidas se han colocado las correas metálicas, espaciadas a $2,10 \mathrm{~m}$, sobre las que se han apoyado las placas de hormigón, prefabricadas, empleadas como elementos de cubrición. Estas correas salvan una luz de 8,50 metros.

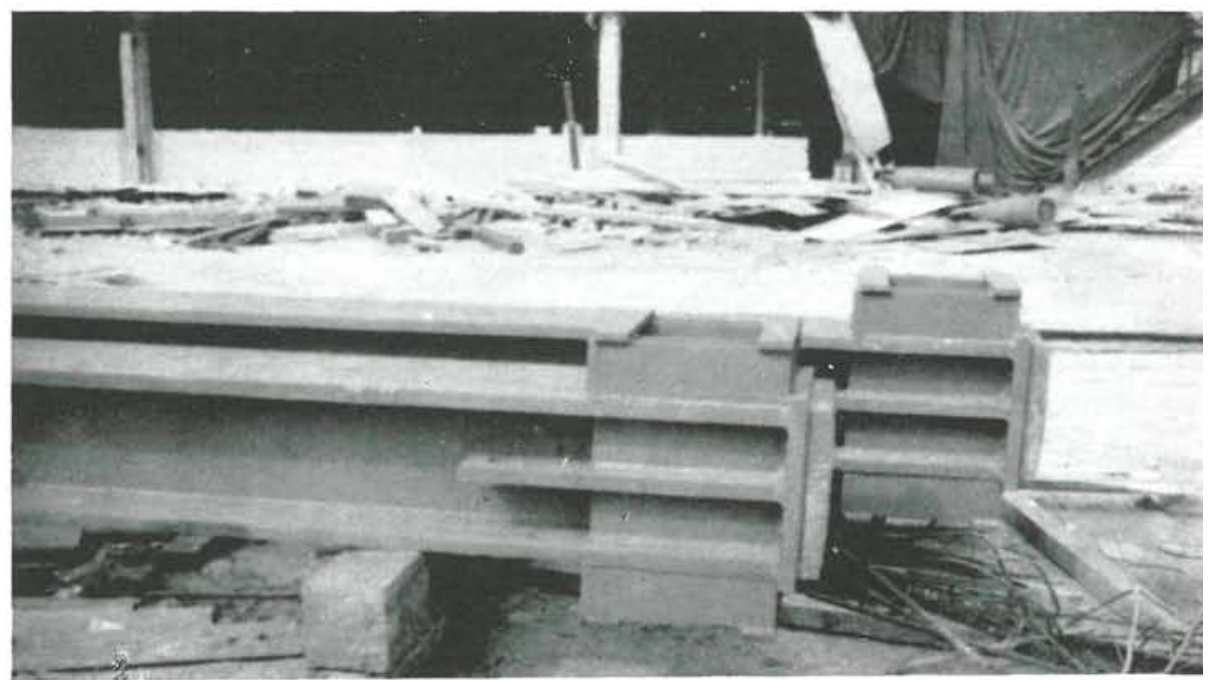

Cables en posición y tesados.

Vista próxima de una silla.

Vista lateral de la base de un mástil. 

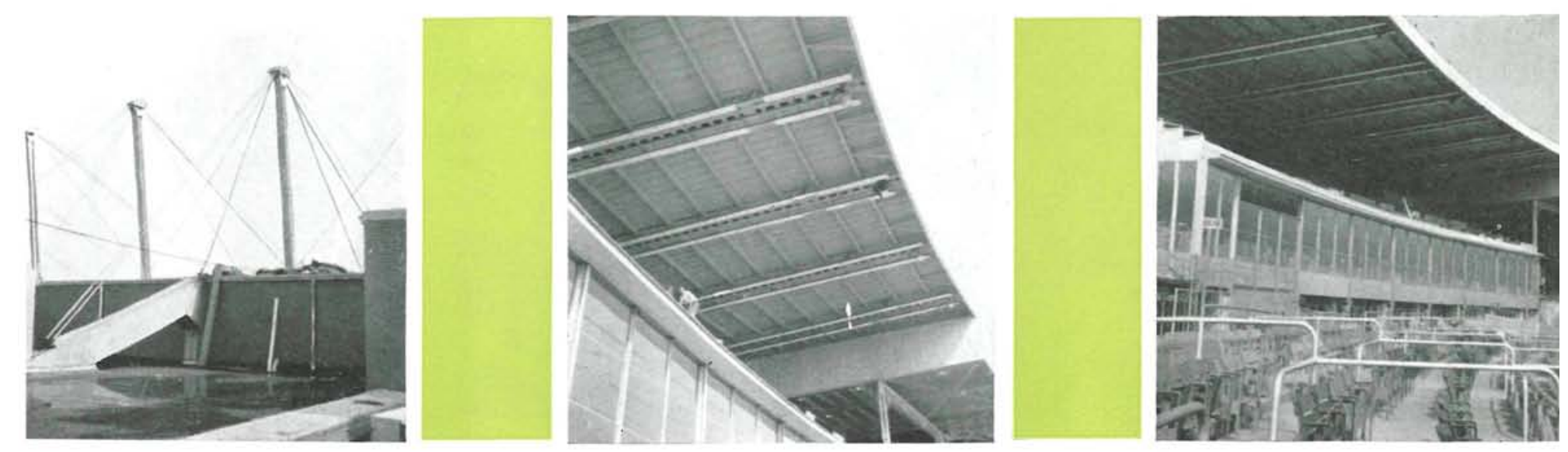

\section{Construcción}

La construcción de la cubierta se inició antes de haber terminado el edificio del cuerpo central, que constituye una estructura de hormigón armado. Cada una de las vigas metálicas, tipo cajón, disponía de dos entramados de andamio o castilletes, situados a la altura de los puntos de amarre con los cables de suspensión. En la parte inferior de estos entramados provisionales se colocaron unos gatos para poder ajustar la altura de suspensión con cierta exactitud.

Las vigas cajón se armaron con perfiles de doble $\mathrm{T}$, número 27, de ala ancha, espaciados a $0,45 \mathrm{~m}$, que se cerraron con placas de $9,5 \mathrm{~mm}$ soldadas en la parte superior e inferior. Cada una de estas piezas pesa más de 10 toneladas, y se transportaron a la obra de una sola longitud, colocándolas en obra mediante grúas móviles provistas de pluma de $33 \mathrm{~m}$. En la parte posterior se apoyaron sobre sus asientos, y en la anterior, sobre los entramados de andamio. Después se colocaron los vientos diagonales de arriostramiento. Cada uno de los mástiles se anclaron en su base por medio de pernos. Hasta después de colocar los cables de suspensión, los mástiles trabajaban como miembros rígidos hasta el momento de colocar los cables de suspensión. Cada viga se suspendió con dos cables amarrados a 4,8 m de su extremidad, y otro cable interior se amarró a $11,80 \mathrm{~m}$ de los otros dos. Los cables se tesaron por medio de tensores de tuerca. Finalmente, se aflojaron los pernos de anclaje de los mástiles para que éstos pudieran pivotar sobre su base. Las operaciones de tesado definitivo se realizaron con gatos hidráulicos de 100 toneladas de capacidad. En primer lugar se puso en carga el cable interior y después los dos exteriores. La carga de los cables interiores es de 30 toneladas, y de 23 para los exteriores o de las extremidades.

El alargamiento calculado para resistir al peso propio fue de $34 \mathrm{~mm}$ en la longitud que separa el mástil a la unión con la viga. El dispositivo de tesado y gatos fue tal, que estas operaciones se pudieron llevar a cabo de una forma continua. Los cálculos se pudieron comprobar en la obra, ya que, al llegar a la carga que corresponde al alargamiento previsto, se empezó a levantar los entramados de las torres provisionales. Los cables se probaron antes de emplearlos en la obra. 


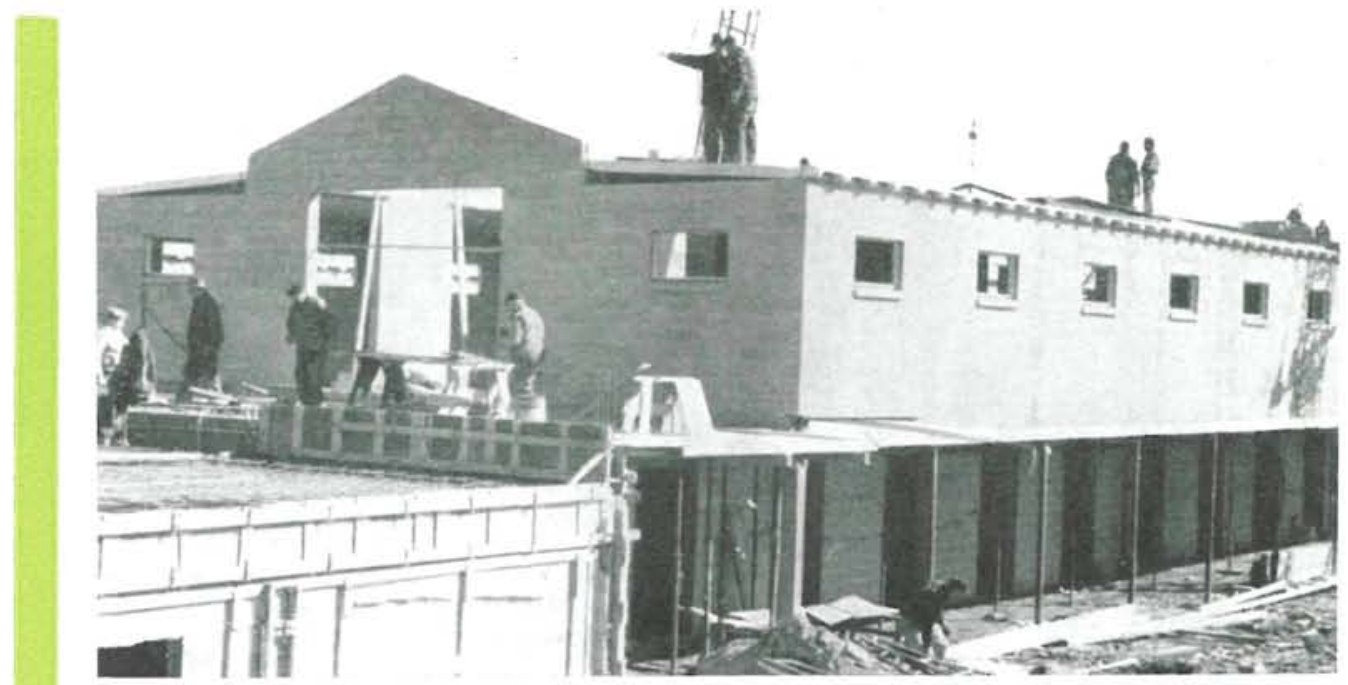

Cuadras en construcción.

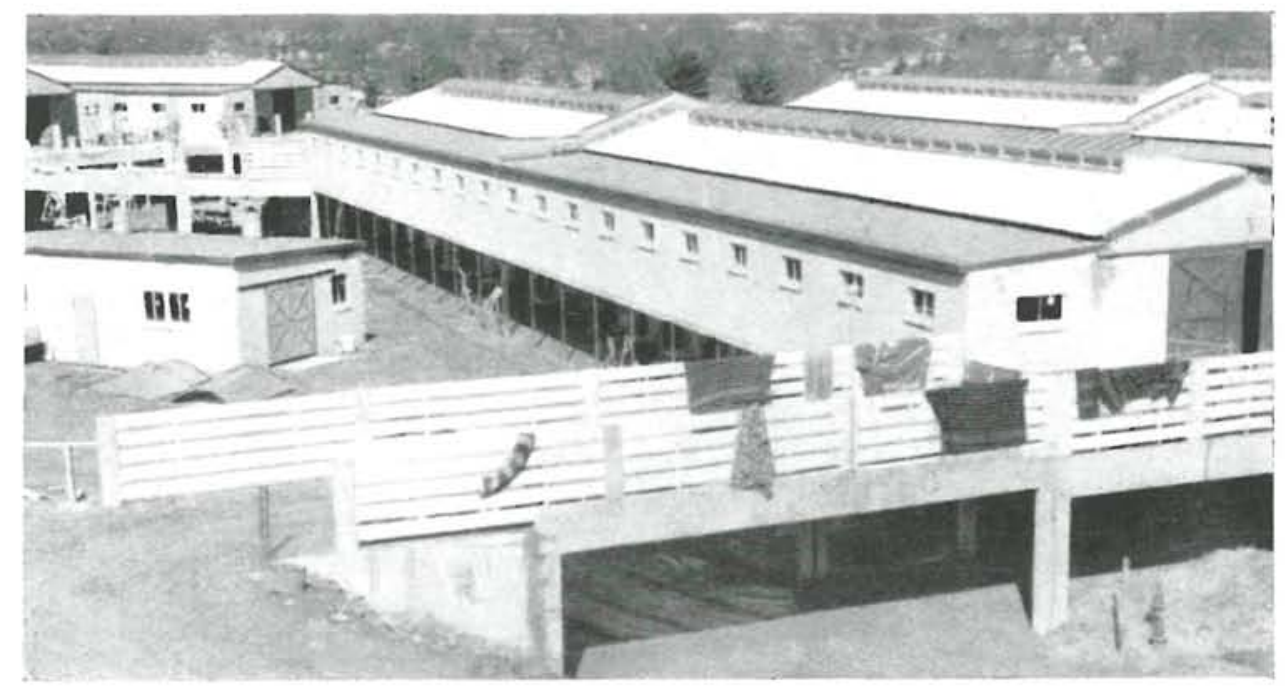

Cuadras terminadas.

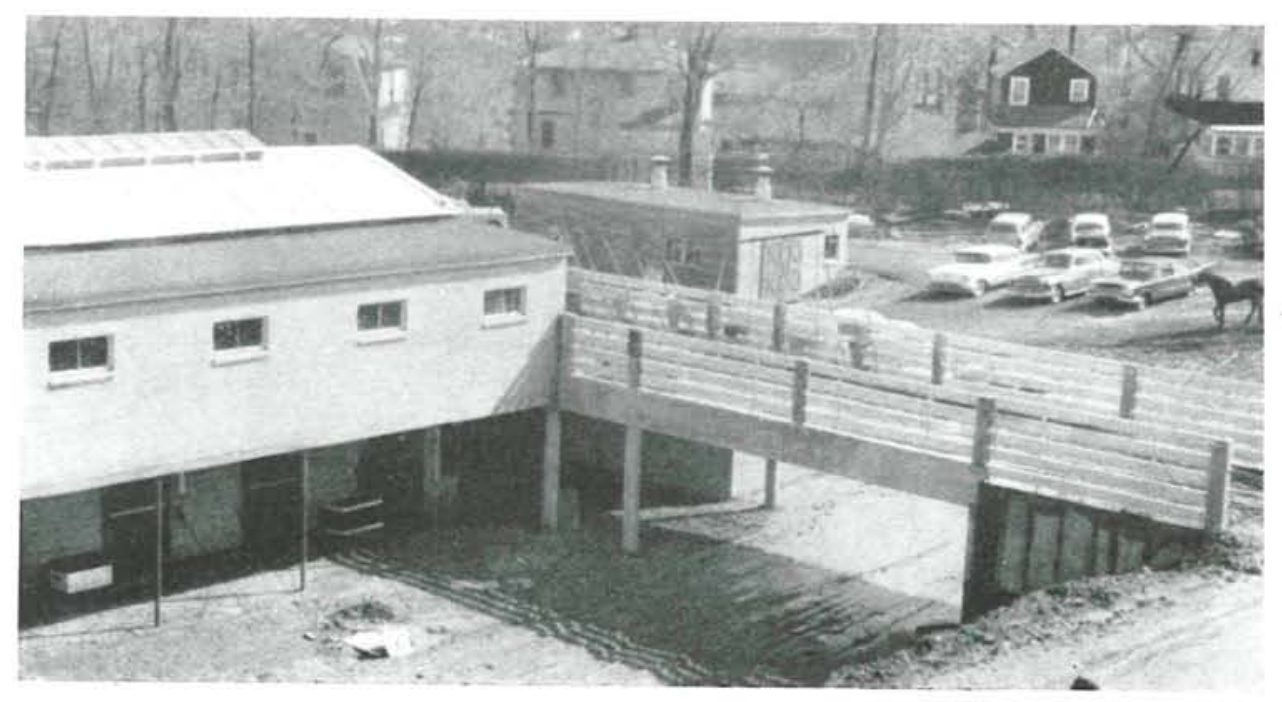

Pontón de paso a las cuadras 
Durante un período de dos años consecutivos se han venido siguiendo todos los movimientos provocados por vientos, nieves, etc., sin que las posiciones de la extremidad de las vigas suspendidas, de $29 \mathrm{~m}$ de longitud en voladizo, hayan variado en más de $2 \mathrm{~cm}$. Para el análisis estático de la estructura mixta de cables y elementos rígidos se empleó el método de energía mínima.

\section{Ideas generales sobre el proyecto}

A excepción de la cubierta, se ha hecho uso del hormigón armado de preferencia. Los soportes son del tipo cilíndrico y zunchados. Las terrazas, formadas con losas de hormigón armado, se apoyan sobre entramados formando pórticos de travesaños inclinados. Las losas que forman las terrazas se han dispuesto formando huella y contra-huella, como si se tratase de una escalera. Estos elementos son del tipo nervado y tienen un espesor mínimo de hormigón de $12 \mathrm{~cm}$. El espesor total de las losas depende del lugar y carga, por lo que es variable. En las proximidades de los soportes se han previsto las armaduras correspondientes para resistir a los esfuerzos cortantes y permitir una buena distribución de
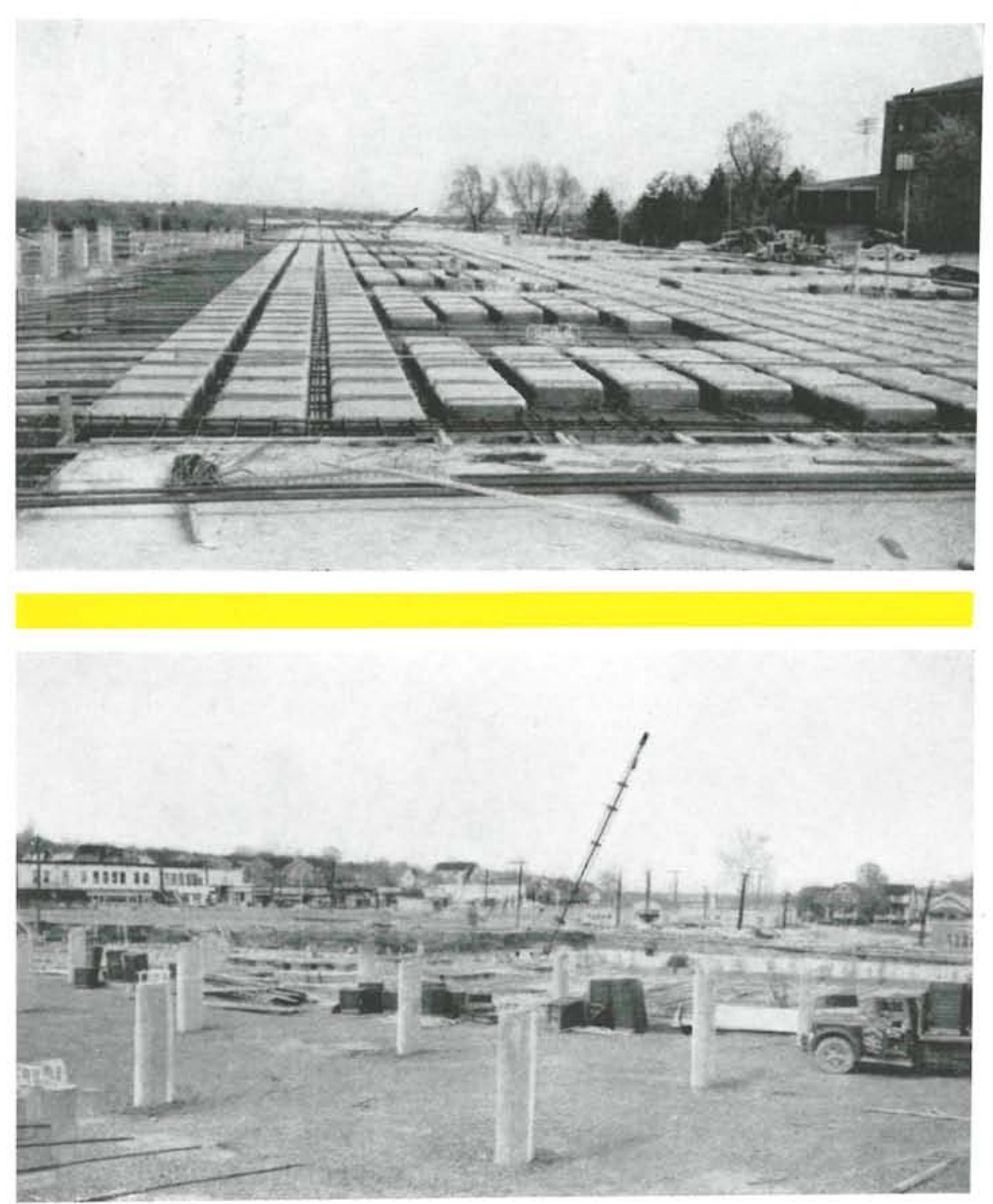
cargas. También se han previsto las armaduras necesarias, en la parte superior del espesor de losa en los soportes, con objeto de hacer frente a la presencia de los momentos negativos que en estas zonas se desarrollan.

La marquesina que cubre la entrada principal del hipódromo, formada por placas plegadas de hormigón, y la lámina que cubre una estación terminal de autobuses, fueron objeto especial de estudio en el proyecto. A partir de esta entrada, los espectadores tienen acceso a cualquier parte del recinto.

\section{Garaje}

Con objeto de habilitar una zona para estacionamiento de vehículos se ha construído un garaje, de cuatro plantas, con capacidad para 3.000 coches, con una rampa de acceso para cada planta, y una general, de salida, de dirección única, a la que convergen los distintos vehículos, que necesitan unos $30 \mathrm{mi}-$ nutos para su evacuación total.

Forjado del garaje.

Soportes del garaje. 


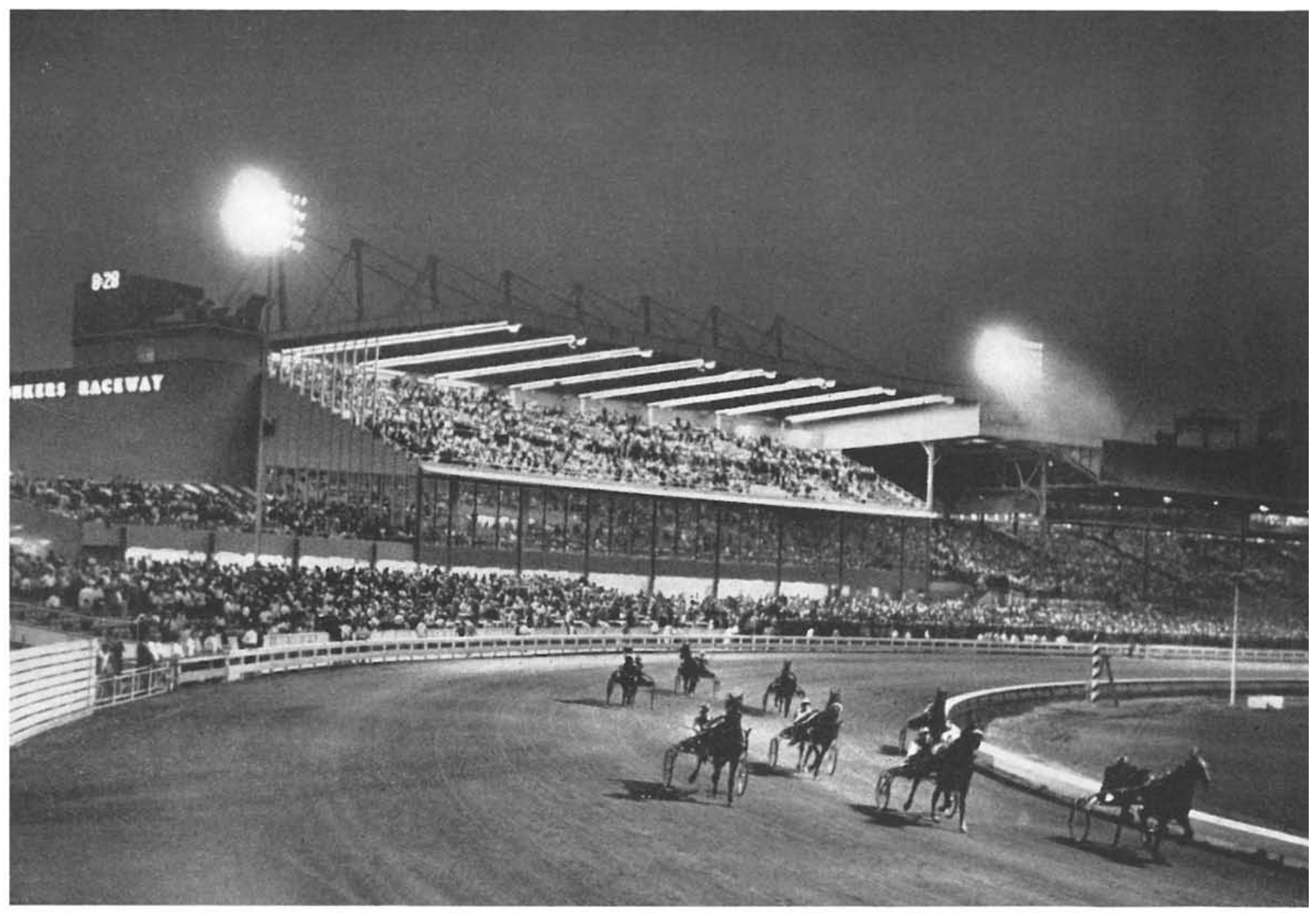

En planta, el garaje tiene $164 \times 170 \mathrm{~m}$, superficie que se ha subdividido en cuatro partes por medio de juntas de dilatación. En la parte superior, las juntas se han utilizado con objeto de disponer drenes en su interior para el desagüe en caso de lluvias. Cada una de estas plantas tiene una pendiente del $1 \%$ en la dirección diagonal, pendiente que se ha utilizado con diferentes finalidades. En el futuro, y en vista de los resultados logrados, esta pendiente se elevará al $2 \%$.

Esta estructura es del tipo corriente, de soportes distribuídos formando rectángulos y forjados para cada planta. Las secciones de los soportes se presentan con vértices redondeados para evitar las rozaduras con los vehículos que estacionan en su interior. 